\title{
Towards a working model for the abundance variations within globular cluster stars
}

\author{
Francesca D'Antona ${ }^{1}$, Paolo Ventura ${ }^{1}$, \\ and Vittoria Caloi ${ }^{2}$ \\ ${ }^{1}$ INAF Osservatorio di Roma, I-00040 Monteporzio (Italy) \\ email: dantona@mporzio.astro.it, ventura@mporzio.astro.it \\ ${ }^{2}$ INAF Istituto di Astrofisica Spaziale, via del Fosso del Cavaliere, 00100 Rona, Italy \\ email: vittoria.caloi@rm.iasf.cnr.it
}

\begin{abstract}
We summarize the results of recent model computation for massive Asymptotic Giant Branch stars of low metallicity, whose winds are thought to be the matter from which second generation stars are born in Globular Clusters (GCs) showing abundance anomalies. The yields of these ejecta are highly uncertain, but our models in the range of masses $3.5-4.5 \mathrm{M}_{\odot}$ can reasonably explain some of the chemical anomalies of Globular Cluster stars.
\end{abstract}

Keywords. Globular clusters, stars: AGB stars and post-AGB, nucleosynthesis

\section{The paradigma: two stages of star formation in GCs}

Fenner et al. (2004) results for the evolution of AGB stars of low metallicity seems to contradict the widely accepted paradigm that the chemical anomalies among GC stars are due to the presence of a second stellar generation born from the ejecta of massive AGB stars. Ventura \& D'Antona (2004) have shown that the results of AGB nucleosynthesis depend dramatically on the assumptions hidden in these models computation, and especially on the convection treatment. The comparison between the results of these two works for the O-Na anticorrelation are shown in the left side of Fig. 1: both sets of models have problems, but at least the evolution of $3.5-4.5 \mathrm{M}_{\odot}$ from Ventura \& D'Antona (2005) with standard mass loss (left bottom line) and mass loss increased by a factor 5 (right bottom line) can consistently explain the anticorrelation. Notice in fact that the models must explain only the stars with high $\mathrm{Na}$ - moderately low Oxygen (central circle in the figure), as the most extreme oxygen depletions are seen only in evolved stars in M13 (left circle) and may be due to an additional evolutionary depletion of oxygen acting during the giant phase. There is, in addition, a fraction of stars with "normal" abundances (right circle). In this light of interpretation we also show how our models deal with the Lithium abundances in NGC 6752 (right Fig. 1, data from Pasquini, this volume). We see that the $4 \mathrm{M}_{\odot}$ model yields are consistent with the slightly smaller Lithium in stars with high sodium: the "quasi normal" lithium in stars with high nitrogen can be explained only as a result of hot bottom burning. Our $5-6 \mathrm{M}_{\odot}$ models, which destroy Na, of course are not consistent with the $\mathrm{Na}-\mathrm{Li}$ anticorrelation. The $\mathrm{CN}$ and $\mathrm{ON}$ data (Cohen \& Melendez, private communication) are reasonably interpreted (Fig. 2).

\section{References}

Fenner,Y., Campbell, S., Karakas, A.I., Lattanzio, J.C. \& Gibson, B.K. 2004, MNRAS, 353, 789 Ventura, P., \& D'Antona, F. 2005, A\&A, 431, 279 

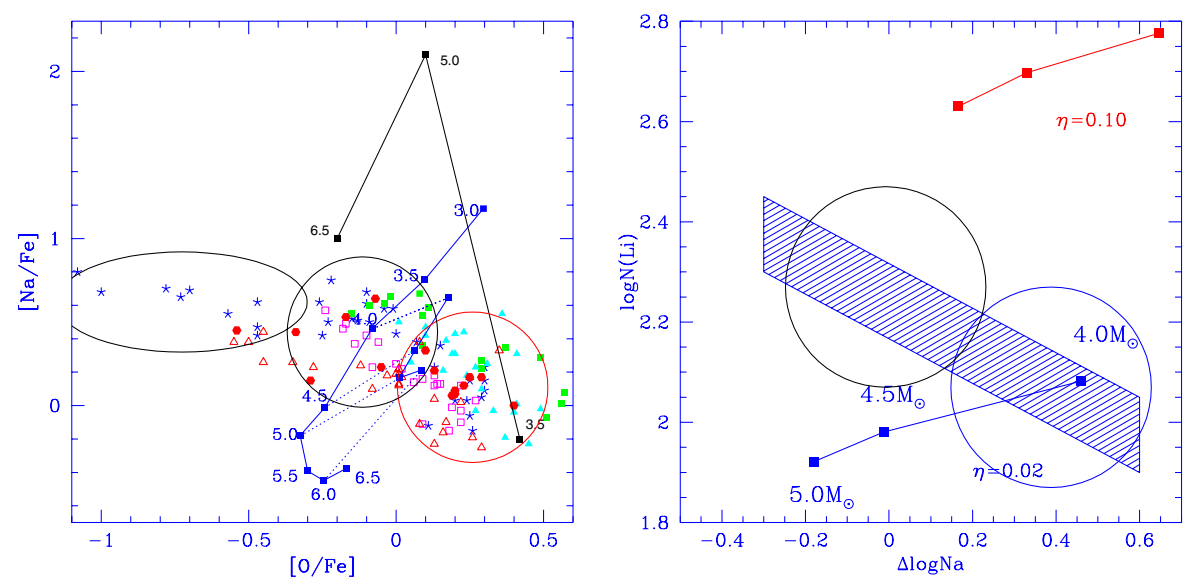

Figure 1. (Left panel): Observed data in the $\mathrm{Na}-\mathrm{O}$ for several GCs from various sources (references in Ventura \& D'Antona 2005). Stars: M13, open squares: M3 (both from Sneden and his group); full squares: NGC 6752 from Grundahl; full triangles: M4 and open triangles: M5 (both from Ivans); full dots: NGC 2808 from Carretta and his group. Models by Fenner et al. (2004) and by Ventura \& D'Antona (2005) are shown. Right panel: locus of Na-Li abundances in NGC 6752 (Pasquini, this volume) and our interpretation: normal abundances of the first generation stars (right circle) and abundances for the second generation (left circle).
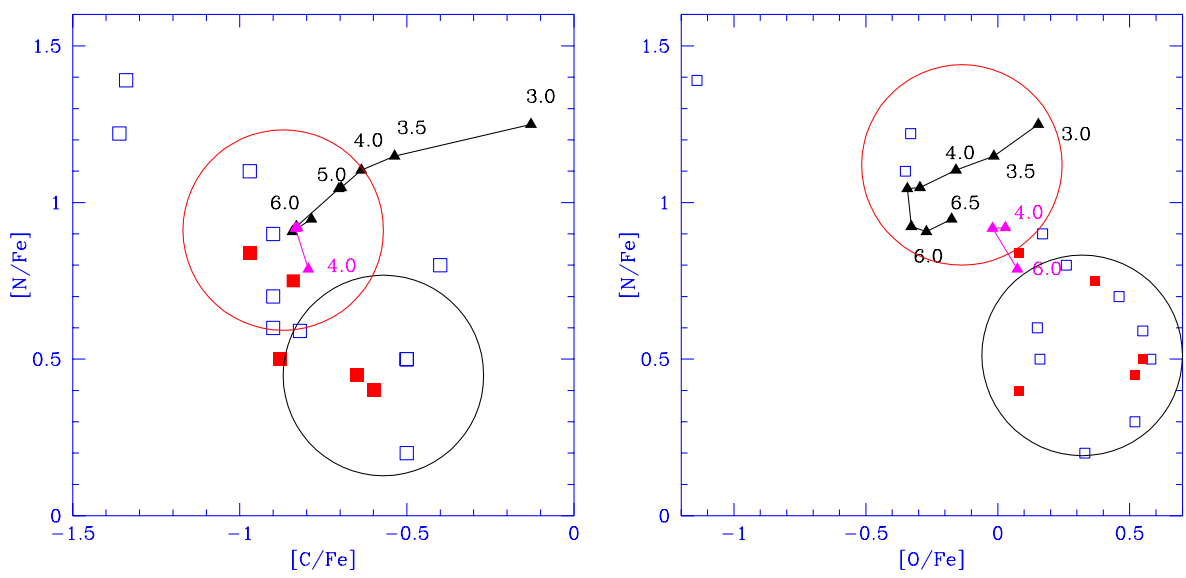

Figure 2. CN (left) and ON (right) in the stars of M3 (full squares) and M13 (open squares) compared with our models. The locus of the normal abundances of the first stellar generation is tenetatively identified as the circles at the left. The data are by courtesy of Melendez and Cohen. 